\title{
HEAT TRANSFER WHILE INCORPORATING METAL PARTICLES IN A MELTING SUPPORT PLATE
}

\author{
Gennady Ya. Mamontov ${ }^{1, *}$, and Danil N. Chuguev \\ ${ }^{1}$ National Research Tomsk Polytechnic University, 634050 Tomsk, Russia \\ ${ }^{2}$ PJSC "LUKOIL"
}

\begin{abstract}
The work defines and solves the heat transfer problem for "particle - support plate" system heated to high temperatures by means of mathematical simulation methods. In the course of the formulated problem, the numerical studies were carried out under conditions of a high density of the particle material in comparison with the support plate material.
\end{abstract}

\section{Introduction}

Over the last few decades, the processes running if different hardening coatings are applied on the surface of mechanical engineering, petroleum chemistry and power engineering equipment structural units have been studied intensively [1]. In these circumstances, various approaches based predominantly on theoretical studies are used [1,2]. But up to the present moment there are no published models that in a unique manner describe the hightemperature interaction mechanisms of a single particle and a support plate surface (that is an element of some equipment) under conditions of a high-temperature impact on the latter one (e.g. while plasma coating). It is hard if not impossible to visualize by experiment an interaction process of a fully or partially melted metal single droplet heated to high temperatures and a support plate (that can be also melted). However, it is known [3] (based on the results of the particle and support plate adhesion zone microstructure analysis) that even under conditions of the particle motion low speed at the impact moment (up to $20 \mathrm{mps}$ ), in numerous cases, the latter are incorporated in a support plate.

One of the possible mechanisms of such incorporation can be a displacement by a particle of the melted metal boundary layer at its contact at the instant of encounter. Furthermore, the energy necessary for penetration of such support plate layer can be released also as a result of the particle metal crystallization. Traditionally, while plasma coating at the instant of encounter, the latter presents a heterogeneous structure - a droplet some part $(20-25 \%)$ of which is crystallized. Until recently, the mathematical models taking into consideration the aforementioned interaction processes of a particle and a support plate under conditions of hardening coating application (at high temperatures) while simulating the main characteristics of hardening plasma technologies of processing equipment structural elements were not in use.

${ }^{*}$ Corresponding author: gmamontov@tpu.ru 
The work objective is a mathematical simulation of the heat transfer while incorporating a metal particle in a support plate that melts at a heating process by means of energy accumulated in the particle and a crystallization heat under conditions of low speed gravity sedimentation.

\section{Problem definition and solution pattern}

A disk-shaped particle positioned at the surface of a metal sheet is considered (fig. 1). The temperature of the metal sheet is lower than that of the particle the bottom part of which ( $10 \%$ from top to bottom) is crystallized. As a result of the particle heat conductivity, a thin layer of the support plate metal is heated to the melting point (or higher) at contact with the heating source (particle) and a thin melt sheet is formed. Under the action of gravity, the particle expels the melt and incorporates into the support plate, which is heated to a full particle crystallization by means of the phase transition heat release. The incorporation speed is determined by the support plate metal fusion penetration. The studied process is transient due to the energy limitedness accumulated in the particle.

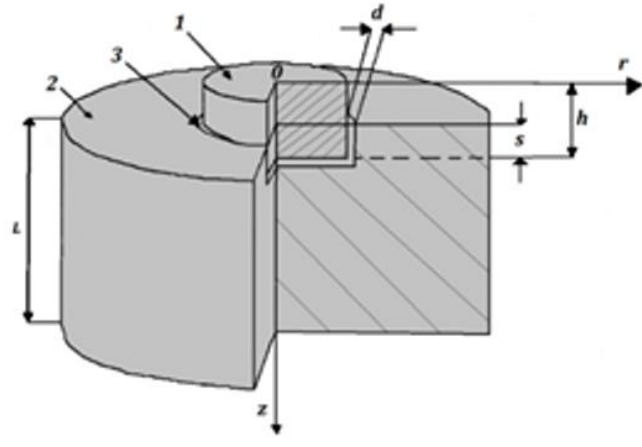

a)

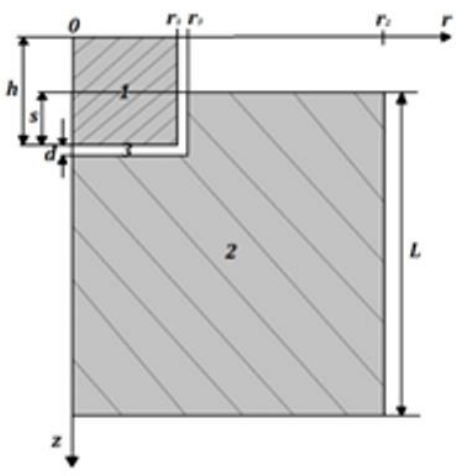

b)

Fig. 1. Overview diagram of the studied process (a). Geometric layout of the process under study (b). 1, 2, 3- particle, support plate and melt area respectively.

While setting a problem it was supposed, in a similar way to [4], that the particle keeps a disk shape throughout the crystallization process; the maximum temperatures reached under conditions of the phase transition do not exceed the melted support plate metal vaporization point; the particle energy is sufficient to incorporate at depth corresponding to a melting point $T_{p}$; the particle advance speed to a depth of the support plate is determined by the isotherm $T=T_{p}$ advance speed on the particle symmetry axis.

To describe the heat transfer processes within the studied "particle - support plate" system, a mathematical model developed to solve condensed material firing problems was used as baseline through the in-situ heating of the single heated to high-temperature particle $[5,6]$.

The nonlinear differential equation system of transient heat conductivity, continuity, motion and energy for the "particle - support plate" system as part of the stated physical analog is as follows:

$$
\begin{aligned}
& \mathrm{c}_{1} \rho_{1} \frac{\partial T_{1}}{\partial t}=\lambda_{1}\left(\frac{\partial^{2} T_{1}}{\partial r^{2}}+\frac{1}{r} \frac{\partial T_{1}}{\partial r}\right)+\lambda_{1} \frac{\partial^{2} T_{1}}{\partial z^{2}}+\frac{Q_{1} W_{1}}{h} \\
& \mathrm{c}_{2} \rho_{2} \frac{\partial T_{2}}{\partial t}=\lambda_{2}\left(\frac{\partial^{2} T_{2}}{\partial r^{2}}+\frac{1}{r} \frac{\partial T_{2}}{\partial r}\right)+\lambda_{2} \frac{\partial^{2} T_{2}}{\partial z^{2}}+\frac{Q_{2} W_{2}}{h}
\end{aligned}
$$




$$
\begin{gathered}
\mathrm{c}_{3} \rho_{3} \frac{\partial T_{3}}{\partial t}=\lambda_{3}\left(\frac{\partial^{2} T_{3}}{\partial r^{2}}+\frac{1}{r} \frac{\partial T_{3}}{\partial r}\right)+\lambda_{3} \frac{\partial^{2} T_{3}}{\partial z^{2}} \\
\frac{\partial u}{\partial r}+\frac{u}{r}+\frac{\partial v}{\partial z}=0 \\
\frac{\partial u}{\partial t}+u \frac{\partial u}{\partial r}+v \frac{\partial u}{\partial z}=-\frac{1}{\rho_{3}} \frac{\partial p}{\partial r}+\mu\left(\frac{\partial^{2} u}{\partial z^{2}}+\frac{1}{r} \frac{\partial u}{\partial r}-\frac{u}{r^{2}}\right. \\
\mathrm{c}_{3} \rho_{3}\left(\frac{\partial T_{3}}{\partial t}+u \frac{\partial T_{3}}{\partial r}+v \frac{\partial T_{3}}{\partial z}\right)=\lambda_{3} \frac{\partial^{2} T_{3}}{\partial z^{2}}+\mu\left(\frac{\partial u}{\partial z}\right)^{2} \\
\frac{\partial}{\partial r}(r u)+\frac{\partial}{\partial z}(r v)=0 \\
\frac{\partial v}{\partial t}+u \frac{\partial v}{\partial r}+v \frac{\partial v}{\partial z}=-\frac{1}{\rho_{3}} \frac{\partial p}{\partial z}+\frac{\mu}{\rho_{3} r} \frac{\partial}{\partial r}\left(r \frac{\partial v}{\partial r}\right)+g \\
\mathrm{c}_{3} \rho_{3}\left(\frac{\partial T_{3}}{\partial t}+u \frac{\partial T_{3}}{\partial r}+v \frac{\partial T_{3}}{\partial z}\right)=\lambda_{3} \frac{\partial^{2} T_{3}}{\partial r^{2}}+\mu\left(\frac{\partial u}{\partial r}\right)^{2}
\end{gathered}
$$

The mass melting and crystallization rate is calculated using the formula:

$$
\begin{gathered}
W_{i}=\rho_{i} V_{i}, \text { where } i=1,2 \\
V_{i}(r, z, t)=\frac{\xi(r, z, t+\Delta t)-\xi(r, z, t)}{\Delta t}
\end{gathered}
$$

Based on prime geometric relationships $V_{i}$ can be viewed as:

$$
\begin{gathered}
V_{i}(r, z, t)=\left[\frac{T(r, 0, t+\Delta t)-T_{m}}{T(r, 0, t+\Delta t)-T(0, h, t+\Delta t)}-\frac{T(r, 0, t)-T_{m}}{T(r, 0, t)-T(0, h, t)}\right] \frac{h}{\Delta t} \\
s=\int_{0}^{t_{k}} V_{2} d t .
\end{gathered}
$$

It is accepted that the particle, support plate and support plate melt area symmetry axes are in line. The particle size $-\left(0 \leq \mathrm{r} \leq \mathrm{r}_{1}\right.$ and $\left.0 \leq \mathrm{z} \leq \mathrm{h}\right)$, support plate $-\left(0 \leq \mathrm{r} \leq \mathrm{r}_{2}\right.$ and $0 \leq \mathrm{z}$ $\leq \mathrm{L})$, melt area $-\left(0 \leq \mathrm{r} \leq \mathrm{r}_{1}, \mathrm{~h} \leq \mathrm{z} \leq \mathrm{h}+\mathrm{d}\right) \cup\left(\mathrm{r}_{1} \leq \mathrm{r} \leq \mathrm{r}_{3}\right.$ and $\left.\mathrm{h}-\mathrm{s} \leq \mathrm{z} \leq \mathrm{h}+\mathrm{d}\right)$.

The (1) - (9) system boundary conditions are written as:

$$
\begin{gathered}
z=0,0 \leq r \leq r_{1}:-\lambda_{1} \frac{\partial T_{1}}{\partial z}=\alpha\left(T_{g}-T_{1}\right)+\sigma \varepsilon_{e m}\left(T_{g}^{4}-T_{1}^{4}\right) \\
r=r_{1}, 0 \leq z \leq h-s:-\lambda_{1} \frac{\partial T_{1}}{\partial r}=\alpha\left(T_{g}-T_{1}\right)+\sigma \varepsilon_{e m}\left(T_{g}^{4}-T_{1}^{4}\right) \\
r=r_{1}, h-s \leq z \leq h:-\lambda_{1} \frac{\partial T_{1}}{\partial r}=-\lambda_{3} \frac{\partial T_{3}}{\partial r}, T_{1}=T_{3} \\
z=h, 0 \leq r \leq r_{1}:-\lambda_{1} \frac{\partial T_{1}}{\partial z}=-\lambda_{3} \frac{\partial T_{3}}{\partial z}, T_{1}=T_{3} \\
r=0,0 \leq z \leq L+h-s:-\lambda_{i} \frac{\partial T_{i}}{\partial r}=0, \text { where } i=1,2,3
\end{gathered}
$$




$$
\begin{gathered}
z=h-s, r_{1} \leq r \leq r_{2}:-\lambda_{i} \frac{\partial T_{i}}{\partial z}=\alpha\left(T_{g}-T_{i}\right)+ \\
\sigma \varepsilon_{e m}\left(T_{g}^{4}-T_{i}^{4}\right), \text { where } i=2,3 \\
r=r_{3}, h-s \leq z \leq h+d:-\lambda_{3} \frac{\partial T_{3}}{\partial r}=-\lambda_{2} \frac{\partial T_{2}}{\partial r}, T_{3}=T_{2} \\
z=h+d, 0 \leq r \leq r_{3}:-\lambda_{3} \frac{\partial T_{3}}{\partial z}=-\lambda_{2} \frac{\partial T_{2}}{\partial z}, T_{3}=T_{2} \\
r=r_{2}, h-s \leq z \leq L+h-s:-\lambda_{2} \frac{\partial T_{2}}{\partial r}=0 \\
z=L+h-s, 0 \leq r \leq r_{2}:-\lambda_{2} \frac{\partial T_{2}}{\partial z}=0 \\
r=0, h \leq z \leq h+d: u=0 \\
z=h, 0 \leq r \leq r_{1}: u=0 \\
r=r_{1}, h-s \leq z \leq h: v=0 \\
r=r_{3}, h-s \leq z \leq h+d: v=0 \\
z=h-s, r_{1} \leq r \leq r_{3}: u=0 \\
z=h+d, 0 \leq r \leq r_{3}: u=U_{m} \\
z=h, r_{1} \leq r \leq r_{3}: v=V_{m}
\end{gathered}
$$

The (1) - (9) system initial conditions are as follows:

$$
\text { where } t=0: T_{1}(r, z)=T_{p}, T_{2}(r, z)=T_{s}, s=0, d=0
$$

The given emissivity factor $\varepsilon_{e m}$ of an object system where the radiant heat transfer is in progress, is calculated using the formula [11]:

$$
\varepsilon_{e m}=\frac{1}{\frac{1}{\varepsilon_{g}}+\frac{1}{\varepsilon_{m}}-1} .
$$

The key agreed notations are: $\mathrm{T}-$ temperature; $\lambda$ - heat conductivity; $\mathrm{c}-$ heat capacity; $\rho$ - density; $r, z$ - cylindrical coordinates; $\mathrm{t}$ - time; $\mathrm{Q}, \mathrm{W}$ - heat effect and phase transition rate; $\mathrm{u}, v$ - melt rate coordinates; $\mu$-dynamic viscosity; $\mathrm{V}$ - linear advance speed of melt and crystallization boundary; h, s, d, L - particle, support plate and hollow space typical dimensions.

The problem of a metal melt flow in a slot hollow space between the particle and a solid support plate part was solved as part of a thin passage model [7].

The stated equation system with the corresponding boundary conditions and additional relations is solved by means of a finite-difference method developed to solve the heat transfer nonlinear problems under conditions of active physical-chemical algorithm transformations $[8,9]$. A nonlinear and unequally spaced difference mesh was used. Owing to the absence of experimental findings and solutions of even very simplified similar 
problems, the numerical simulation result reliability was assessed by means of numerical solutions based on condensing grid consecutive order.

\section{Findings and discussions}

The numerical studies within the limits of the formulated problem were carried under conditions of a higher particle material density as opposed to the support plate metal. With such ratio of densities, we meet the particle incorporation condition only due to gravitation work that is stronger than the lift force acting on the particle on the side of the melt. To demonstrate more clearly the studied process conformity, the density $\rho_{1} / \rho_{2}$ ratio was as great as practicable in the real world - 7.8.

Figure 2 displays the distinctive positions of typical isotherms while incorporating the particle in a melted support plate part. It can be seen that the melt sheet bed depth reaches $0.001 \mathrm{~m}$. under the particle and near the flat side. The distinctive positions of typical isotherms and field velocities in a vertical and horizontal part of the hollow space (fig. 1) are presented in figure 3 . The studied process conformity analysis was carried out.

It is determined that if the particle is considered as a metal droplet which is melted at the contact moment with the support plate, in the course of crystallization the metal layer, which is the front side adjacent to the particle, freezes at rapid-fire pace even under relatively low $(200-250 \mathrm{~K})$ heat overloads in relation to the support plate. On further motion, the particle is made as a disk the front side of which is solid and the remaining part is melted.

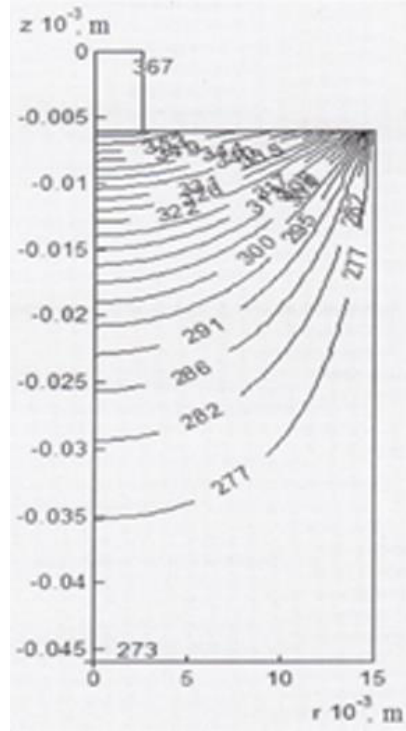

a)

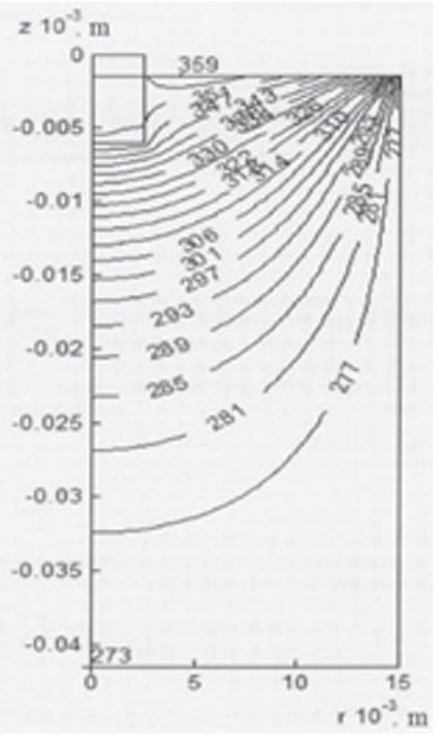

b)

Fig. 2. The typical isotherm position while incorporating a metal particle ( $\mathrm{a}$ - initial instant, $\mathrm{b}-$ finite time instant).

The numerical simulation results give grounds for conclusion that if the particle has a certain kinetic energy store in case of sedimentation on the support plate and is solid, it will incorporate in the support plate without any postheating due to the heat transition phase. In this case, the incorporation depth will be determined by the impact velocity and deformation (virtual) of the particle. Thus, the ratio of characteristic heat time, particle deformation, support plate fusion penetration and particle incorporation is essential. 


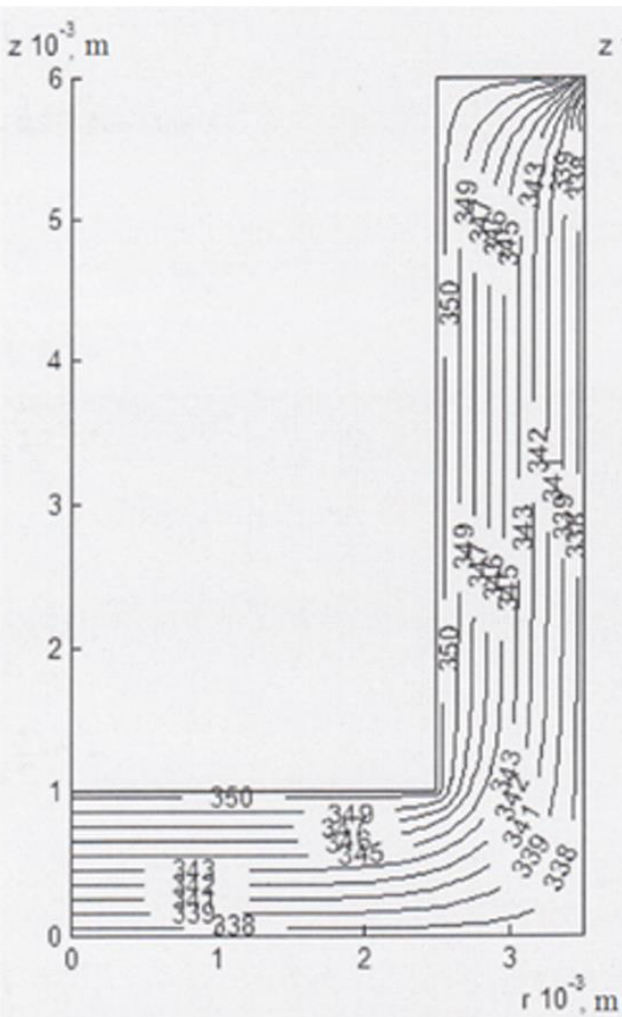

a)

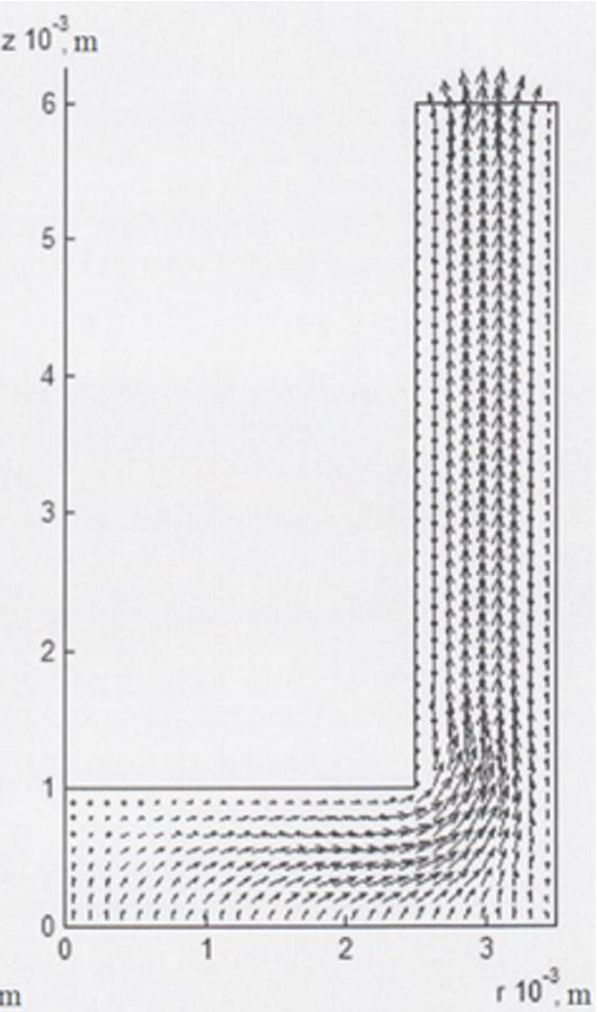

b)

Fig. 3. Typical isotherm position (a) and field velocities (b) in a vertical and horizontal passage. $(\mathrm{Re}=$ 100).

The numerical study results showed that the beneficial significant effect could be obtained by means of the support plate pre-heating to temperatures close to the material melting point.

\section{Conclusion}

As a result of the heat conductivity problem solution for "heated up to high temperature particle - melting support plate" system, the possibility to incorporate the particle in a material three-dimensional layer of the hardenable detail within the limits of the basic process characteristic change that corresponds to coating technologies, was determined. The formulated mathematical model and the heat transfer problem solution method can be used to select technological processes for hardening and protecting coating application on the surface of machine and device components.

\section{Acknowledgments}

The work was supported by the Russian President's grant (Scientific School project 7538.2016.8) 


\section{References}

1. Yu.A. Chumakov, A.G. Knyazeva, Combust. Explos. Shock Waves 48 (2012)

2. E.M. Ivanov, A.A. Uglov, Physics and chemistry of material processing 2 (1985)

3. A.M. Dubasov, V.V. Kudinov, M.X. Shorshov, Physics and chemistry of material processing 6 (1971)

4. G.V. Kuznetsov, G.Ya Mamontov, G.V. Taratushkina, Combust. Explos. Shock Waves 40, 1 (2004)

5. G.V. Kuznetsov, G.Ya. Mamontov, G.V. Taratushkina, Khimicheskaya Fizika 23, 3 (2004)

6. G.V. Kuznetsov, P.A. Strizhak, J. Eng. Thermophys. 17 (2008)

7. P. J. Roache, Computational Fluid Dynamics, Hermosa Publishers, Albuquerque (NM, USA, 1976)

8. V.L. Strakhov, A.N. Garashchenko, G.V. Kuznetsov, V.P. Rudzinskii, Combust. Explos. Shock Waves 37 (2001)

9. O.V. Vysokomornaya, G.V. Kuznetsov, P.A. Strizhak, Russ. J. Phys. Chem. B 5 (2011) 